ISSN 0103-9954

\title{
DIVERSIDADE DE FORMIGAS (HYMENOPTERA: FORMICIDAE) DA SERRAPILHEIRA EM DIFERENTES SISTEMAS DE USO DO SOLO
}

\section{DIVERSITY OF ANTS (HYMENOPTERA: FORMICIDAE) OF LITTER IN DIFFERENT SYSTEMS OF SOIL USE}

\author{
Edison Bisognin Cantarelli ${ }^{1}$ Marciane Danniela Fleck ${ }^{2}$ Fabiane Granzotto ${ }^{3}$ \\ Janaína De Nadai Corassa ${ }^{4}$ Marcia d'Avila ${ }^{5}$
}

\begin{abstract}
RESUMO
Neste trabalho são listadas as espécies de formigas de serrapilheira encontradas em quatro ambientes localizados na região noroeste do estado do Rio Grande do Sul, a fim de verificar o impacto da ação antrópica realizada no meio rural. Para a coleta das formigas da serrapilheira foi utilizado o método do extrator de Winkler. Foram coletados 6.300 espécimes pertencentes a oito subfamílias, 18 tribos, 31 gêneros e 108 espécies. A floresta nativa possui a maior riqueza observada com 90 espécies coletadas, seguida por eucalipto com 65 espécies, agricultura e pastagem exótica com 20 espécies cada. O índice de diversidade de Margalef foi de 11,21 para área com floresta nativa, 8,37 para eucalipto, 3,48 para agricultura e, 2,71 na área de pastagem exótica. O índice de diversidade de Shannon obtido foi de 2,89; 3,15;2,43 e 1,98 e de equitabilidade 0,$64 ; 0,75 ; 0,84$ e 0,66 para as áreas com: floresta nativa, eucalipto, agricultura e pastagem, respectivamente. O maior valor do índice de diversidade para a área de eucalipto pode ser devido à área possuir 28 anos de implantação, sem ter sido manejada, com sub-bosque de espécies nativas, formando um dossel contínuo e espessa camada de serrapilheira, já a floresta nativa por sofrer intervenções antrópicas, possui um valor de diversidade de Shannon menor comparado à área com eucalipto.
\end{abstract}

Palavras-chave: riqueza; bioindicador; extrator mini-Winkler.

\begin{abstract}
Litter ant species found in four sites located in northwestern Rio Grande do Sul state were listed in this study to verify the impact of human activities conducted in rural areas. The method used for the collection of litter ants was the Winkler extractor. There were collected 6,300 specimens belonging to eight subfamilies, 18 tribes, 31 genera and 108 species. Native forest presented the highest richness observed with 90 species collected, followed by 65 eucalyptus species, and agriculture and pasture with 20 exotic species each. Margalef's diversity index was 11.21 for area with native forest, 8.37 for eucalypt, 3.48 for agriculture, and 2.71 in exotic pasture area. The Shannon's diversity indices obtained were 2.89, 3.15, 2.43 and 1.98 and equitability indices of $0.64,0.75,0.84$ and 0.66 for areas with native forest, eucalyptus, agriculture and

1 Engenheiro Florestal, Dr., Professor Associado IV do Departamento de Engenharia Florestal, Centro de Educação Superior Norte do Rio Grande do Sul, Universidade Federal de Santa Maria, BR386, Km 40, Linha 7 de Setembro, CEP 98400-000, Frederico Westphalen (RS), Brasil. cantarelli@ufsm.br

2 Engenheira Florestal, Doutoranda do Programa de Pós-graduação em Engenharia Florestal, Centro de Ciências Rurais, Universidade Federal de Santa Maria, Av. Roraima, 1000, CEP 97105-000, Santa Maria (RS), Brasil. marcianedanniela@gmail.com

3 Engenheira Florestal, Especialista em Gestão Ambiental, Mestranda do Programa de Pós-graduação em Engenharia de Processos, Centro de Tecnologia, Universidade Federal de Santa Maria, Av. Roraima, 1000, CEP 97105-000, Santa Maria (RS), Brasil.fabianegranzotto@yahoo.com.br

4 Engenheira Florestal, Dr ${ }^{\mathrm{a}}$., Professora Adjunta, Instituto de Ciências Agrárias e Ambientais, Universidade Federal de Mato Grosso, Av. Alexandre Ferronato, 1200, Setor Industrial, Campus Universitário, CEP 78557-267, Sinop (MT), Brasil. janadenadai@gmail.com

5 Engenheira Florestal, Dr $^{\mathrm{a}}$., Professora Adjunta do Departamento de Engenharia Florestal, Centro de Educação Superior Norte do Rio Grande do Sul, Universidade Federal de Santa Maria, BR386, Km 40, Linha 7 de Setembro, CEP 98400-000, Frederico Westphalen (RS), Brasil. davilamar@hotmail.com
\end{abstract}

Recebido para publicação em 27/09/2011 e aceito em 21/10/2013

Ci. Fl., v. 25, n. 3, jul.-set., 2015 
exotic pasture, respectively. The highest diversity index for the eucalypt area may be due to the age of the forest (28 years) and the fact that it has not been managed as well as due to the presence of understory of native species, forming a continuous canopy and thick litter layer. On the other hand, since the native forest has suffered human interventions, it has a lower Shannon's diversity index compared to the eucalypt area. Keywords: wealth; bio-indicator; extractor mini-Winkler.

\section{INTRODUÇÃO}

O impacto negativo que a espécie humana exerce sobre o ambiente, como a expansão do uso da terra, tem levado à necessidade de desenvolver conceitos e estabelecer novos critérios sobre as formas de uso dos recursos ambientais (DIEHL et al., 2005). Um dos conceitos é o de uso sustentável, o qual, segundo Campanhola (2001), necessariamente envolve a interação de três componentes: o biológico, o econômico e o social.

No estado do Rio Grande do Sul, a partir de 1872, iniciou-se, de forma contínua, uma corrente imigratória destinada à ocupação de um extenso território desabitado, à mercê dos países vizinhos e à produção de gêneros para o mercado urbano que começava a se constituir (VACCARO, 2002).

As atividades humanas, cada vez mais intensas, têm causado inúmeras alterações ambientais, muitas colocando em risco a vida silvestre, de forma que estudos sobre biodiversidade são prementes (WILSON, 1997; MCGEOCH e CHOWN, 1998). Por suas características biológicas e ecológicas, as formigas (MAJER, 1983; EGGLETON et al., 1995; ANDERSEN, 1997; SILVA e BRANDÃO, 1999) têm sido referidas como importantes organismos indicadores da biodiversidade (DIEHL, 2009). Em florestas, especialmente nas tropicais, a riqueza de espécies de formigas é alta, mas tende a diminuir com os desmatamentos. Essa redução geralmente é acompanhada pela perda seletiva de alguns grupos funcionais, assim como pelo aumento relativo de outros (EGGLETON et al., 1995).

As formigas constituem um dos grupos de insetos mais conhecidos e estudados (HÖLLDOBLER e WILSON, 1990), sendo consideradas como um dos principais componentes biológicos de ambientes estruturalmente complexos como as florestas (FITTKAU e KLINGE, 1973). São boas indicadoras do estado de conservação dos ecossistemas terrestres, por possuírem riqueza de espécies local e global altas, facilidades para serem amostradas padronizadamente, possibilidade de identificar as espécies, importância ecológica e respostas rápidas aos estresses ambientais, bem como pelo fato de terem sua diversidade relacionada com vários outros componentes bióticos (MAJER, 1983; BRANDÃO, 1999).

Neste sentido, este trabalho teve por objetivo conhecer e avaliar sistemas tradicionais de uso do solo no noroeste do Rio Grande do Sul, na região do Alto Uruguai, como eucalipto, agricultura e pastagem exótica, comparados com a floresta nativa, para avaliar a diversidade dos formicídeos presentes nos ambientes. Em cada ambiente objetivou-se determinar a composição, a riqueza de Margalef, a diversidade, a equitabilidade e a riqueza observada das espécies de formigas, a fim de verificar qual atividade antrópica realizada no meio rural concilia produção com menor impacto ambiental.

\section{MATERIAL E MÉTODO}

\section{Caracterização do local de estudo}

O trabalho foi desenvolvido em quatro áreas próximas entre si, porém, não contíguas, no município de Frederico Westphalen - RS $\left(27^{\circ} 21^{\prime} 33^{\prime \prime}\right.$ S e $\left.53^{\circ} 23^{\prime} 40^{\prime \prime} \mathrm{W}\right)$, em região inserida no bioma Mata Atlântica, onde predominam as formações do tipo Floresta Estacional Decidual. Estes ambientes são constituídos por: 1) floresta nativa com 15 ha e aproximadamente 60 anos de idade com relatos de supressão de árvores, presença de clareiras e intervenções antrópicas na área; 2) plantio de Eucalyptus spp. com 1 ha e 28 anos de idade, sem espaçamento determinado com sub-bosque de vegetação nativa diversificada; 3) agricultura com 3 ha e 30 anos de idade, com presença de milho (Zea mays L.); 4) pastagem exótica constituído de gramíneas da família Poaceae: Eragrostis plana Nees; Cynodon dactylon (L.) Pers.; Andropogon bicornis L.; Setaria perviflora (Poir.) Kerguélen; Axonopus compressus (Sw.) P. Beauv. e Paspalum distichum Linn., possui 2 ha e 10 anos de idade. 


\section{Coleta de dados}

A coleta de formigas foi realizada durante seis meses (setembro/2008-fevereiro/2009), na área da Universidade Federal de Santa Maria campus de Frederico Westphalen - RS. Em cada ambiente foram efetuadas coletas mensais. Nas áreas de floresta nativa, eucalipto e pastagem exótica, as coletas foram realizadas de setembro a janeiro. $\mathrm{Na}$ área de agricultura, buscou-se amostrar a fauna de formigas em todo o ciclo do cultivo do milho. Assim, a primeira coleta foi realizada quando as plantas estavam com, aproximadamente, $10 \mathrm{~cm}$ de altura e nas duas coletas posteriores, as mesmas apresentavam $30 \mathrm{~cm}$ de altura. As duas últimas coletas foram realizadas após a colheita do milho, em janeiro e fevereiro de 2009. A colheita do milho ocorreu de forma manual, restando na área todo o material vegetal seco.

Em cada área foram efetuadas cinco coletas, sendo amostrados quatro pontos com três amostras cada, com distância mínima de 20 metros da borda do sistema e 10 metros entre si. As três amostras de cada ponto foram misturadas resultando em uma amostra para cada ponto. Em cada amostra foi retirada a serrapilheira contida em $1 \mathrm{~m}^{2}$ até o aparecimento da superfície do solo, sendo que o volume das amostras variou entre os pontos. Depois de peneiradas, as amostras de serrapilheira foram transferidas para os extratores de mini-Winkler, nos quais permaneceram por $72 \mathrm{~h}$, para a extração das formigas. Ao final das cinco coletas, cada área analisada possuía 20 amostras (cinco coletas em quatro pontos amostrais).

As formigas foram triadas e separadas dos demais artrópodes, sendo armazenadas em frascos com álcool 70\%, devidamente etiquetados, para posterior identificação. Com o auxílio de microscópio estereoscópico, as formigas foram separadas por amostras e enviadas para especialistas do Laboratório de Mirmecologia do Centro de Pesquisas do Cacau/Comissão Executiva do Plano da Lavoura Cacaueira (CEPEC/CEPLAC), Ilhéus - BA, que procederam à identificação dos exemplares em nível de espécie.

Séries das espécies coletadas foram etiquetadas e depositadas nas coleções do laboratório de Proteção Florestal, da Universidade Federal de Santa Maria campus de Frederico Westphalen, assim como os demais artrópodes.

\section{Análise dos dados}

A análise dos dados foi realizada utilizandose medidas de diversidade pelo método de Margalef e Shannon e o índice de equitabilidade, através do programa Anafau (MORAES et al., 2003). A riqueza observada foi obtida pelo número absoluto de espécies de formigas coletadas pelo método do extrator de Winkler em cada ambiente.

\section{RESULTADOS E DISCUSSÃO}

Foram coletados 6.300 espécimes de formigas, distribuídos em 108 espécies, 31 gêneros, 18 tribos e oito subfamílias. Os gêneros com o maior número de espécies foram Pheidole com 26 espécies e Camponotus, Solenopsis e Hypoponera com oito espécies cada (Tabela 1).

$\mathrm{Na}$ área de floresta nativa foram capturadas 90 espécies e na área de eucalipto 65 espécies de formigas. A área de agricultura e a área de pastagem exótica apresentaram ambas, 20 espécies de formigas, $78 \%$ menos espécies em comparação com a floresta nativa, refletindo a importância da floresta nativa como um reservatório da fauna de formigas e de outros invertebrados (LUTINSKI et al., 2008).

A área de plantio de eucalipto apresenta-se estruturalmente similar à floresta nativa, apesar de se tratar de um sistema monoespecífico, devido ao porte arbóreo da espécie, com 28 anos de implantação, presença de sub-bosque e grande quantidade de serrapilheira, resultado que corrobora com os encontrados por Braga et al. (2010). Esse fato influencia tanto na diversidade, quanto na composição de espécies de formigas presentes no agroecossistema, aproximando-o da floresta nativa. Por outro lado, a agricultura e a pastagem exótica apresentam-se como agroecossistemas completamente distintos dos demais ambientes analisados no que diz respeito à riqueza de formigas.

A subfamília com o maior número de táxons foi Myrmicinae, com 10 tribos, 18 gêneros e 66 espécies corroborando os resultados de Feitosa e Ribeiro (2005) e Santos et al. (2006), que, utilizando o mesmo método de coleta, registraram a subfamília Myrmicinae com maior número de espécies. Soares et al. (1998), Schmidt et al. (2005), Diehl et al. (2005), Silva e Silvestre (2000), embora utilizando métodos de coleta e ambientes diferentes, obtiveram resultado similar.

Isso ocorre certamente porque a 
TABELA 1: Registros das espécies de formigas coletadas em áreas de floresta nativa, eucalipto, agricultura e pastagem exótica. Frederico Westphalen - RS. Setembro de 2008 a fevereiro de 2009.

TABLE 1: Records of ant species collected in areas of native forest, eucalyptus, exotic pasture and agriculture. Frederico Westphalen, RS state. September 2008 to February 2009.

\begin{tabular}{|c|c|c|c|c|}
\hline \multirow{2}{*}{ Subfamília/Tribo/Espécies } & \multicolumn{4}{|c|}{ Espécimes } \\
\hline & Floresta Nativa & Eucalipto & Agricultura & Pastagem exótica \\
\hline \multicolumn{5}{|l|}{ Subfamília Amblyoponinae } \\
\hline \multicolumn{5}{|l|}{ Tribo Amblyoponini } \\
\hline Amblyopone armigera Mayr & 1 & - & - & - \\
\hline Amblyopone elongata (Santschi) & 7 & - & - & - \\
\hline \multicolumn{5}{|l|}{ Subfamília Dolichoderinae } \\
\hline \multicolumn{5}{|l|}{ Tribo Dolichoderini } \\
\hline Dorymyrmex thoracicus Gallardo & 4 & - & - & - \\
\hline Linepithema humile (Mayr) & 4 & - & - & - \\
\hline Linepithema sp.1 & - & 1 & - & - \\
\hline Tapinoma sp.1 & 4 & 6 & - & - \\
\hline \multicolumn{5}{|l|}{ Subfamília Ectatomminae } \\
\hline \multicolumn{5}{|l|}{ Tribo Ectatommini } \\
\hline Gnamptogenys moelleri (Forel) & 1 & - & - & - \\
\hline Gnamptogenys striatula Mayr & 133 & - & - & - \\
\hline \multicolumn{5}{|l|}{ Subfamília Formicinae } \\
\hline \multicolumn{5}{|l|}{ Tribo Camponotini } \\
\hline Camponotus crassus Mayr & 1 & 22 & 4 & - \\
\hline Camponotus (Myrmaphaenus) sp.1 & 1 & - & - & - \\
\hline Camponotus arboreus (Fr. Smith) & 3 & 18 & - & - \\
\hline Camponotus fastigatus Roger & - & 54 & - & - \\
\hline Camponotus melanoticus Emery & 9 & 39 & 3 & - \\
\hline Camponotus rufipes (Fabricius) & 12 & 37 & - & - \\
\hline Camponotus sericeiventris (Guérin) & 1 & - & - & - \\
\hline Camponotus sp.1 & - & 2 & - & - \\
\hline \multicolumn{5}{|l|}{ Tribo Plagiolepidini } \\
\hline Brachymyrmex heeri Forel & 2 & 32 & - & - \\
\hline Brachymyrmex sp.1 & 2 & 1 & 1 & - \\
\hline Brachymyrmex sp. 2 & 1 & - & - & - \\
\hline Brachymyrmex sp. 3 & - & 2 & - & - \\
\hline Paratrechina longicornis (Latreille) & 132 & 29 & 74 & 374 \\
\hline Paratrechina sp.1 & 36 & 166 & - & 166 \\
\hline Paratrechina sp. 2 & 2 & 2 & - & 123 \\
\hline Paratrechina sp. 3 & - & 81 & - & - \\
\hline Paratrechina sp.4 & - & 8 & - & - \\
\hline Paratrechina (Nylanderia) sp. 5 & 6 & - & - & - \\
\hline \multicolumn{5}{|l|}{ Subfamília Heteroponerinae } \\
\hline \multicolumn{5}{|l|}{ Tribo Heteroponerini } \\
\hline Heteroponera dolo (Roger) & 3 & - & - & - \\
\hline Heteroponera mayri Kempf & 18 & - & - & - \\
\hline Heteroponera sp.1 & 1 & - & - & - \\
\hline Subfamília Myrmicinae & & & & \\
\hline
\end{tabular}

Continua.. 
TABELA 1: Continuação...

TABLE 1: Continued...

\begin{tabular}{|c|c|c|c|c|}
\hline \multirow{2}{*}{ Subfamília/Tribo/Espécies } & \multicolumn{4}{|c|}{ Espécimes } \\
\hline & Floresta Nativa & Eucalipto & Agricultura & Pastagem exótica \\
\hline \multicolumn{5}{|l|}{ Tribo Attini } \\
\hline Acromyrmex laticeps Emery & 7 & 93 & - & \\
\hline Acromyrmex lundi (Guérin) & 4 & - & - & - \\
\hline Acromyrmex sp. 1 & - & 5 & - & - \\
\hline Apterostigma madidiense Weber & 58 & 2 & - & - \\
\hline Apterostigma sp. complexo pilosum Mayr & 1 & - & - & - \\
\hline Apterostigma sp.1 & 1 & - & - & - \\
\hline Atta sexdens piriventris Santschi & 32 & 1 & - & 1 \\
\hline Cyphomyrmex major Forel & 2 & - & - & - \\
\hline Cyphomyrmex sp.1 & 1 & - & - & - \\
\hline Cyphomyrmex sp. 2 & - & 55 & - & - \\
\hline Cyphomyrmex transversus Emery & 1 & 2 & - & - \\
\hline Cyphomyrmex vorticis Weber & 3 & 28 & - & - \\
\hline Myrmicocrypta sp.1 & 5 & - & - & - \\
\hline Trachymyrmex sp.1 & - & 1 & - & - \\
\hline \multicolumn{5}{|l|}{ Tribo Basicerotini } \\
\hline Basiceros discigera (Mayr) & 13 & 4 & - & - \\
\hline Basiceros sp.1 & 1 & - & - & - \\
\hline Octostruma rugifera (Mayr) & 14 & 24 & - & - \\
\hline Octostruma stenognatha Brown e Kempf & 25 & 90 & - & - \\
\hline \multicolumn{5}{|l|}{ Tribo Blepharidattini } \\
\hline Wasmannia auropunctata (Roger) & 4 & - & - & 5 \\
\hline Wasmannia rochai Forel & 2 & - & - & - \\
\hline \multicolumn{5}{|l|}{ Tribo Cephalotini } \\
\hline Cephalotes pusillus (Klug) & 1 & - & - & - \\
\hline \multicolumn{5}{|l|}{ Tribo Crematogastrini } \\
\hline Crematogaster sp.1 & 7 & - & 4 & 51 \\
\hline Crematogaster sp.2 & - & - & 1 & - \\
\hline \multicolumn{5}{|l|}{ Tribo Dacetini } \\
\hline Acanthognathus rudis Brown e Kempf & 1 & - & - & - \\
\hline Strumigenys (Pyramica) rugithorax (Kempf) & 1 & - & - & - \\
\hline Strumigenys cosmotela Kempf & 1 & - & - & - \\
\hline Strumigenys silvestrii Emery & 39 & 105 & 9 & 2 \\
\hline \multicolumn{5}{|l|}{ Tribo Myrmicini } \\
\hline Hylomyrma balzani (Emery) & 159 & 21 & - & - \\
\hline Hylomyrma sp.1 & 1 & 6 & - & - \\
\hline \multicolumn{5}{|l|}{ Tribo Pheidolini } \\
\hline Pheidole aper Forel & 10 & 3 & - & - \\
\hline Pheidole heyeri Forel & 5 & 19 & - & - \\
\hline Pheidole obscurithorax Naves & 4 & 1 & - & - \\
\hline Pheidole radoszkoowskii Mayr & 100 & 84 & - & - \\
\hline Pheidole rugatula Santschi & 54 & 92 & - & 6 \\
\hline Pheidole siggilata Wilson & 38 & 34 & 29 & - \\
\hline
\end{tabular}

Continua... 
TABELA 1: Continuação...

TABLE 1: Continued...

\begin{tabular}{|c|c|c|c|c|}
\hline \multirow{2}{*}{ Subfamília/Tribo/Espécies } & \multicolumn{4}{|c|}{ Espécimes } \\
\hline & Floresta Nativa & Eucalipto & Agricultura & Pastagem exótica \\
\hline Pheidole senilis Santschi & 1 & - & - & - \\
\hline Pheidole sospes Forel & 58 & 6 & - & - \\
\hline Pheidole sp.1 & 18 & 13 & 110 & - \\
\hline Pheidole sp.2 & 10 & 1 & 19 & 1 \\
\hline Pheidole sp. 3 & 12 & 9 & 4 & 2 \\
\hline Pheidole sp.4 & 9 & 6 & 13 & 1 \\
\hline Pheidole sp.5 & 2 & 2 & - & - \\
\hline Pheidole sp.6 & 1 & - & - & - \\
\hline Pheidole sp.7 & 1 & - & - & - \\
\hline Pheidole sp. 8 & 1 & - & - & - \\
\hline Pheidole sp.9 & 1 & 1 & - & - \\
\hline Pheidole sp.10 & 3 & 2 & - & - \\
\hline Pheidole sp.11 & - & 1 & - & - \\
\hline Pheidole sp.12 & - & 3 & - & - \\
\hline Pheidole sp.13 & - & 1 & - & - \\
\hline Pheidole sp.14 & - & 1 & - & - \\
\hline Pheidole sp.15 & - & 1 & - & - \\
\hline Pheidole sp.16 & 1 & - & - & - \\
\hline Pheidole sp 17 grupo fallax & - & - & - & 118 \\
\hline Pheidole subarmata Mayr & 3 & 15 & 1 & - \\
\hline \multicolumn{5}{|l|}{ Tribo Solenopsidini } \\
\hline Megalomyrmex drifti Kempf & 7 & 19 & - & - \\
\hline Megalomyrmex pusillus Forel & - & 5 & - & - \\
\hline Solenopsis (Diplorhoptrum) sp.1 & 16 & 7 & - & 1 \\
\hline Solenopsis richteri Forel & 125 & 2 & - & 157 \\
\hline Solenopsis saevissima (Fr. Smith) & 876 & 419 & 8 & 40 \\
\hline Solenopsis sp.1 & 5 & - & 1 & - \\
\hline Solenopsis sp.2 & - & - & 11 & - \\
\hline Solenopsis sp. 3 & 38 & - & - & - \\
\hline Solenopsis sp.4 & 1 & - & - & - \\
\hline Solenopsis sp.5 & 3 & - & - & - \\
\hline \multicolumn{5}{|l|}{ Tribo Tetramoriini } \\
\hline Tetramorium bicarinatum Nylander & 9 & 15 & 1 & 1 \\
\hline \multicolumn{5}{|l|}{ Subfamília Ponerinae } \\
\hline \multicolumn{5}{|l|}{ Tribo Ponerini } \\
\hline Anochetus altisquamis Mayr & 13 & - & - & - \\
\hline Hypoponera foreli (Mayr) & 1 & - & - & - \\
\hline Hypoponera opaciceps (Mayr) & 22 & 1 & 16 & - \\
\hline Hypoponera sp.1 & 340 & 230 & 5 & 33 \\
\hline Hypoponera sp.2 & 5 & 9 & - & 3 \\
\hline Hypoponera sp.3 & 80 & 65 & - & 14 \\
\hline Hypoponera sp.4 & 82 & 28 & - & 4 \\
\hline Hypoponera sp.5 & 2 & - & - & - \\
\hline
\end{tabular}

Continua...

Ci. Fl., v. 25, n. 3, jul.-set., 2015 
TABELA 1: Continuação...

TABLE 1: Continued...

\begin{tabular}{lccc}
\hline \multicolumn{1}{c}{ Subfamília/Tribo/Espécies } & \multicolumn{2}{c}{ Espécimes } \\
\cline { 2 - 4 } & Floresta Nativa & Eucalipto & Agricultura \\
\hline Hypoponera sp.6 & 19 & 1 & - \\
Pachycondyla ferruginea (Fr. Smith) & 1 & - & - \\
Pachycondyla harpax (Fabricius) & 12 & 32 & 2 \\
Pachycondyla striata Fr. Smith & 31 & 19 & - \\
Subfamília Proceratiinae & & & - \\
Tribo Proceratiini & & 8 & - \\
Discothyrea sexarticulata Borgmeier & 1 & 21 & 10 \\
\hline Total de gêneros & 30 & 65 & 20 \\
\hline Total de espécies & 90 & 2092 & 316 \\
\hline Total de espécimes & 2789 & 20 \\
\hline
\end{tabular}

subfamília Myrmicinae é a maior subfamília e a mais diversificada em termos locais e mundiais (BRANDÃO, 1999) e possui adaptações ecológicas de todos os tipos (HÖLLDOBLER e WILSON, 1990; CAETANO et al., 2002) quando se trata de hábitos alimentares e nidificação (FOWLER et al., 1991).

Um total de 37 espécies incidiu somente na área de floresta nativa, perfazendo a maior fauna exclusiva dentre as quatro áreas estudadas, sendo: Acanthognathus rudis Brown e Kempf, Acromyrmex lundi (Guérin), Amblyopone armigera Mayr, Amblyopone elongata (Santschi), Anochetus altisquamis Mayr, Apterostigma sp. complexo pilosum Mayr, Apterostigma sp.1, Basiceros sp.1, Brachymyrmex sp.2, Camponotus (Myrmaphaenus) sp.1, Camponotus sericeiventris (Guérin), Cephalotes pusillus (Klug), Cyphomyrmex major Forel, Cyphomyrmex sp.1, Dorymyrmex thoracicus Gallardo, Gnamptogenys moelleri (Forel), Gnamptogenys striatula Mayr, Heteroponera dolo (Roger), Heteroponera mayri Kempf, Heteroponera sp.1, Hypoponera foreli (Mayr), Hypoponera sp.5, Linepithema humile (Mayr), Myrmicocrypta sp.1, Paratrechina (Nylanderia) sp.1, Pachycondyla ferruginea (Fr. Smith), Pheidole senilis Santschi, Pheidole sp.6, Pheidole sp.7, Pheidole sp.8, Pheidole sp.16, Solenopsis sp.3, Solenopsis. sp.4, Solenopsis sp.5, Strumigenys (Pyramica) rugithorax (Kempf), Strumigenys cosmotela Kempf e Wasmannia rochai Forel.

O número expressivo de espécies pode ser explicado pelas condições de preservação das características originais da vegetação, que ainda perduram na área, pelos recursos disponíveis e pelas relações entre a fauna e a flora.

$\mathrm{Na}$ floresta nativa, as cinco espécies mais abundantes em indivíduos em ordem decrescente, foram: Solenopsis saevissima (Fr. Smith), Hypoponera sp.1, Hylomyrma balzani (Emery), Gnamptogenys striatula Mayr e Paratrechina longicornis (Latreille).

A riqueza observada (Figura 1) apresenta tendência de acúmulo crescente com o aumento do esforço amostral. A não estabilização da curva do coletor, para comunidades de formigas, é evento comum e pode estar ligado à distribuição agregada das espécies, quanto também à raridade de várias espécies (SANTOS et al., 2006).

A riqueza de espécies de formigas está correlacionada com a complexidade estrutural do ambiente. Pois, níveis mais elevados de perturbação resultam em uma diminuição na riqueza de espécies e em um aumento na abundância de formigas (VASCONCELOS, 1998).

Dean e Milton (1995) salientaram que a diversidade de insetos está positivamente associada com a diversidade de plantas perenes e negativamente associada com a diversidade de plantas anuais. Então, espera-se que ambientes de maior complexidade estrutural apresentem maior riqueza de espécies, como foi encontrado na área de floresta nativa que possui recursos em que as formigas ocupam os nichos presentes na área.

$\mathrm{O}$ índice de diversidade de Margalef, que procura compensar os efeitos de amostragem dividindo o número de espécies registradas pelo número total de indivíduos na amostra, foi de 11,21 


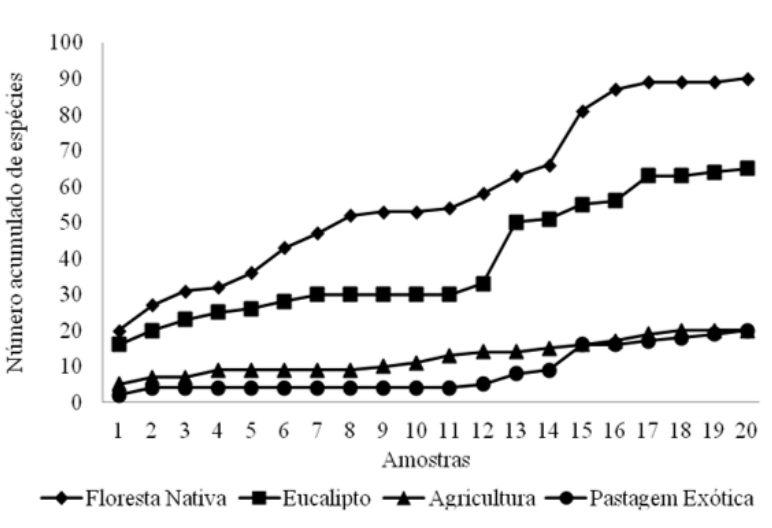

FIGURA 1: Curva de acumulação de espécies de formigas, por unidade amostral, em serrapilheira em quatro ambientes, Frederico Westphalen - RS. Setembro de 2008 a fevereiro de 2009.

FIGURE 1: Accumulation curve of ant species per unit sampling, the litter in four environments, Frederico Westphalen, RS state. September 2008 to February 2009.

para área com floresta nativa, 8,37 para eucalipto, 3,48 para agricultura e, 2,71 na área de pastagem exótica.

O índice de diversidade de Shannon foi de 2,$89 ; 3,15 ; 2,43$ e 1,98 e a equitabilidade foi de 0,$64 ; 0,75 ; 0,84$ e 0,66 para as áreas de floresta nativa, eucalipto, agricultura e pastagem exótica, respectivamente.

SegundoSilveira Neto etal.(1976), em locais em que os fatores limitantes atuam intensamente e a competição interespecífica também, o índice de diversidade tende a diminuir, devido ao aumento do número de espécies mais comuns com grande número de indivíduos e diminuição das espécies mais raras.

O baixo índice de diversidade de Shannon, para a área de pastagem exótica, pode ter ocorrido porque na área há poucos recursos alimentares, além do constante pisoteio do gado. Já na agricultura há uma rotação de espécies, de milho e soja durante o ano, além das espécies de forrageiras no inverno, que podem favorecer e dar condições para uma diversidade maior comparada à pastagem exótica, pois os restos de culturas são incorporados ao solo quando são realizados os plantios.

Como uma medida de heterogeneidade, a equitabilidade leva em consideração o grau de uniformidade na abundância de espécies (MAGURRAN, 2011), então a floresta nativa possui o menor valor de equitabilidade, pois apresenta espécies com muitos indivíduos como Solenopsis saevissima (Fr. Smith) que possui 876 indivíduos, e muitas espécies com poucos indivíduos, ou somente um indivíduo coletado.

Os valores observados para as quatro áreas analisadas sugerem distribuição não uniforme da abundância, ocorrendo forte dominância de algumas espécies, pois, de acordo com Magurran (1988), o índice de equitabilidade varia de 0 a 1 .

Segundo Pinto-Coelho (2000), o índice de equitabilidade é considerado indicativo de uniformidade na distribuição das espécies no local avaliado e esse equilíbrio contribui positivamente para o estabelecimento de uma comunidade de formigas mais diversificada (LUTINSKI et al., 2008).

\section{CONCLUSÕES}

A floresta nativa é mais rica em espécies, apesar de ter sofrido ação antrópica, seguida por plantio de eucalipto.

Myrmicinae é a subfamília de Formicidae com maior diversidade e riqueza nos quatro ambientes analisados.

O plantio de eucalipto, com 28 anos de implantação, é considerado um sistema de uso do solo que causa menos impacto no equilíbrio de formicídeos de serrapilheira, comparado à agricultura e pastagem exótica, no município de Frederico Westphalen - RS.

\section{AGRADECIMENTOS}

Ao pesquisador Jacques Hubert Charles Delabie do Laboratório de Mirmecologia do Centro de Pesquisas do Cacau/Comissão Executiva do Plano da Lavoura Cacaueira (CEPEC/CEPLAC), Ilhéus - BA, pela identificação das espécies de formigas.

\section{REFERÊNCIAS BIBLIOGRÁFICAS}

ANDERSEN, A. N. Using ants as bioindicators: multiscale issues in ant community ecology. Conserve. Ecol. 1997. Disponível em <http: //www. consecol.org/vol1/iss1/ant.8>. Acesso em: 20 de setembro de 2011.

BRAGA, D. L. et al. Avaliação rápida da diver- 
sidade de formigas em sistemas de uso do solo no sul da Bahia. Neotropical Entomology, v. 39, n. 4, p. 464-469, 2010.

BRANDÃO, C. R. F. Família Formicidae. São Paulo, 1999. Disponível em < http:// www. biota.org.br/pdf/v5cap21.pdf >. Acesso em: 22 mar. 2011.

CAETANO, F. H. et al. Formigas: biologia e anatomia. Rio Claro: F.H.C., 2002. 42 p.

CAMPANHOLA, C. Processos e pressões antrópicas que degradam a biodiversidade: estudos de casos. p. 89 - 91. In: GARAY, I.; DIAS, B. (Eds.). Conservação da biodiversidade em ecossistemas tropicais. Petrópolis: Vozes, 2001. 432 p.

DEAN, W. R. J.; MILTON, S. J. Plant and invertebrate assemblages on old fields in the arid southern Karoo, South Africa. African Journal of Ecology, v. 33, p. 1-13, 1995.

DIEHL, E. et al. Riqueza de formigas de solo na praia da Pedreira, Parque Estadual de Itapuã, Viamão, RS, Brasil. Revista Brasileira de Entomologia, v. 49, n. 4, p. 552-556, 2005.

DIEHL, E. Cupins e formigas em remanescentes de Floresta com Araucária, p. 221-228. In: FONSECA, C. R. et al. (Eds.). Floresta com Araucária: Ecologia, Conservação e Desenvolvimento Sustentável. Ribeirão Preto: Holos, 2009.

EGGLETON, P. et al. The species richness of termites (Isoptera) under differing levels of forest disturbance in the Mbalmayo Forest Reserve, southern Cameroon. Journal of Tropical Ecology, v. 11, p. 85-98, 1995.

FEITOSA, R. dos S. M.; RIBEIRO, A. S. Mirmecofauna (Hymenoptera, Formicidae) de serapilheira de uma área de Floresta Atlântica no parque estadual da Cantareira, São Paulo, Brasil. Biotemas, v. 18, n. 2, p. $51-71,2005$.

FITTKAU, E. J.; KLINGE, H. On biomass and trophic structure of the Central Amazonian rain forest Ecosystem. Biotropica, Oxford, v. 5, n. 1, p. 2-14, 1973.

FOWLER, H. G. et al. Ecologia Nutricional de formigas. In: PANIZZI, A. R.; PARRA, J. R. P. (Eds.). Ecologia nutricional de insetos e suas implicações no manejo de pragas. São Paulo: Manole, 1991.

HÖLlDOBLER, B.; WILSON, E. O. The Ants. Massachusetts: Harvard University Press, 1990. $732 \mathrm{p}$.

LUTINSKI, J. A. et al. Diversidade de formigas na Floresta Nacional de Chapecó, Santa Catarina, Brasil. Ciência rural, Santa Maria, v. 38, n. 7, p. 1810-1816, out. 2008.

MAGURRAN, A. E. Medindo a diversidade biológica. Curitiba: Ed. UFPR, 2011. 261p.

MAGURRAN, A. E. Ecological diversity and its measurement. Princeton: Princeton University Press, 1988, $167 \mathrm{p}$.

MAJER, J. D. Ants: Bio-indicators of minesite rehabilitation, land-use, and land conservation. Environmental Management, v. 7, n. 4, p. 375-383, 1983.

MCGEOCH, M. A.; CHOWN, S. L. Scaling up the value of bioindicators. Tree, v. 13, n. 2, p. 46-47, 1998.

MORAES, R.C.B.et al. Software para análise estatística - ANAFAU. In: SIMPÓSIO DE CONTROLE BIOLÓGICO, 8., 2003, São Pedro, SP. Resumos... Piracicaba: ESALQ/USP, 2003, p. 195.

PINTO-COELHO, R. M. Fundamentos em ecologia. Porto Alegre. Artmed, 2000. 252 p.

SANTOS, M. S. et al. Riqueza de formigas (Hymenoptera, Formicidae) da serapilheira em fragmentos de floresta semidecídua da Mata Atlântica na região do Alto do Rio Grande, MG, Brasil. Iheringia, Série Zoologia, Porto Alegre, v. 96, n. 1, p. 95-101, mar. 2006.

SCHMIDT, K. et al. Formigas (Hymenoptera: Formicidae) da Ilha João da Cunha, SC: composição e diversidade. Biotemas, v. 18, n. 1, p. 57-71, 2005.

SILVA, R. R. da; BRANDÃO, C. R. F. Formigas (Hymenoptera: Formicidae) como indicadoras da qualidade ambiental e da diversidade de outros invertebrados terrestres. Biotemas, v. 12, n. 2, p. 55-73, 1999.

SILVA, R. R. da; SILVESTRE, R. Diversidade de formigas (Hymenoptera: Formicidae) em Seara, oeste de Santa Catarina. Biotemas, v. 13, n. 2, p. $85-105,2000$.

SILVEIRA NETO, S. et al. Manual de ecologia de insetos. Piracicaba-SP: Ceres, 1976. $419 \mathrm{p}$.

SOARES, S. M. et al. Diversidade de invertebrados edáficos em áreas de eucalipto e mata secundária. Acta Biol. Leopol. v. 19, p. 157-164, 1998.

VACCARO, S. Crescimento de uma floresta estacional decidual, em três estágios sucessionais, no município de Santa Tereza, RS, Brasil. 2002. $157 \mathrm{f}$. Tese (Doutorado em Engenharia Florestal) Universidade Federal de Santa Maria, Santa Maria, 
2002.

VASCONCELOS, H. L. Respostas das formigas à fragmentação florestal. Série Técnica IPEF, Piracicaba, v. 12, p. 95-98, 1998.
WILSON, E. O. A situação da diversidade biológica. In: WILSON, E. O.; PETER, F. M. (Eds.). Biodiversidade. Rio de Janeiro: Nova Fronteira, 1997, p. 3-24. 